\title{
Clinoptilolita natural mexicana tratada con plata para remover DDD en agua
}

\author{
Mexican natural clinoptilolite treated with silver for DDD removal from water
}

\begin{abstract}
Juana Alvarado-Ibarra',2*, Karla Terán-Samaniego², Diana Vargas-Hernández ${ }^{1,3}$, Ana Laura Bautista-Olivas ${ }^{4}$
Universidad de Sonora, Departamento de Investigación en Polímeros y Materiales. Boulevard Luis Encinas s/n, col. Centro, Hermosillo, Sonora, México. 83000.

2 Universidad de Sonora, Departamento de Ingeniería Industrial. Posgrado en Sustentabilidad. Boulevard Luis Encinas s/n, col. Centro, Hermosillo, Sonora, México. 83000.

3 Cátedra del Consejo Nacional de Ciencia y Tecnología.

${ }^{4}$ Universidad de Sonora, Departamento de Agricultura y Ganadería. Carretera a Bahía Kino, kilómetro 21, Hermosillo, Sonora, México.
\end{abstract}

\section{RESUMEN}

Se estudió el efecto de la temperatura y de la variación de la concentración de plata en una clinoptilolita natural para remover DDD. Mediante las técnicas MEB-EDX y BET se analizó a la muestra natural y modificada. En las pruebas cinéticas se encontró que el equilibrio se presenta a los 180 minutos de contacto y que la plata incrementa la eficiencia de remoción. El proceso presenta una cinética por quimisorción, ya que se ajustó al modelo de pseudo segundo orden y los coeficientes de determinación variaron en un intervalo de 0.9933 a 0.9997. Por las isotermas de adsorción se obtuvo que la máxima concentración adsorbida es de $0.19 \mathrm{mg} / \mathrm{g}$ y los datos ajustaron perfectamente al modelo de Langmuir, en el cual se observa que la constante $b$, relacionada con la energía de adsorción, disminuye cuando hay aumento en la temperatura, lo que indica un decremento de la adsorción a altas temperaturas, teniendo que el orden de adsorción es $25^{\circ} \mathrm{C}>35^{\circ} \mathrm{C}>45^{\circ} \mathrm{C}$. Se concluye que el proceso es de naturaleza exotérmica, al tener que la adsorción es mayor cuando la temperatura es menor, corroborado por el cálculo de los parámetros termodinámicos $\left(\Delta \mathrm{H}^{\circ}, \Delta \mathrm{G}^{\circ}\right.$ y $\left.\Delta \mathrm{S}^{\circ}\right)$.

Palabras clave: clinoptilolita natural, cinética de remoción, isotermas de adsorción, diclorodifenil dicloroetano, plata.

\section{ABSTRACT}

The capability of a natural clinoptilolite to remove DDD in function of the temperature and variation of silver concentration was studied. The MEB-EDX and BET techniques analyzed the natural and modified samples. By kinetic tests, it was found that equilibrium occurred at 180 minutes of contact and that silver increases the removal efficiency. The process displayed a chemisorption kinetic, since it was adjusted to the pseudo second-order model and the coefficients of determination varied in a range from 0.9933 to 0.9997 . By the adsorption isotherms, the maximum adsorbed concentration was obtained at $0.19 \mathrm{mg} / \mathrm{g}$. The data fit perfectly to the Langmuir model, in which a decrease was observed in constant $b$, related to the adsorption energy, when there was an increase in temperature. The latter indicates a decrease in adsorption at high temperatures, with the order of adsorption being $25^{\circ} \mathrm{C}>35^{\circ} \mathrm{C}>45^{\circ} \mathrm{C}$. It is concluded that the process is exothermic, since the adsorption was greater when the temperature lowered, corroborated by the calculation of the thermodynamic parameters $\left(\Delta \mathrm{H}^{\circ}, \Delta \mathrm{G}^{\circ}\right.$ and $\left.\Delta \mathrm{S}^{\circ}\right)$.

Keywords: natural clinoptilolite, kinetic of removal, adsorption isotherms, dichlorodiphenyl dichloroethane, silver.

\section{INTRODUCCIÓN}

El DDD [2,2-Bis (4-clorofenil)-1,1-dicloroetano] no está presente de manera natural en el ambiente (ATSDR, 2016); no obstante, desde el año 1943 éste se ha encontrado en diversos ecosistemas debido a que es un metabolito que surge por la degradación del dicloro difenil tricloroetano (DDT) (Venier e Hites, 2014; Gaspar et al., 2016) que fue utilizado como pesticida desde que se sintetizó en 1939 (Turusov et al., 2002). En los años 1960 se tuvo evidencia que el DDT y sus derivados eran altamente persistentes en el ambiente, tóxicos y que pueden bioacumularse en los grandes mamíferos (Ziv, 2009; Thomas y Gohil, 2011), por lo que fue prohibido su uso en el mundo (Polanco et al., 2015; Bajwa et al., 2016). A pesar de la prohibición, el DDT y el DDD siguen siendo utilizados en diferentes países (ATSDR, 2016), tanto en la agricultura como en programas relacionados con la salud humana, debido a su bajo costo y alta efectividad contra algunos vectores (Zhou et al., 2013). En México, dichas sustancias son de uso restringido, establecido en el catálogo de la Comisión Intersecretarial para el Control del Proceso y uso de Plaguicidas y Sustancias Tóxicas (CICOPLAFEST) (Castro y Díaz, 2004).

Estos pesticidas se han encontrado en el suelo en cantidades considerables 30 años después de haber sido utilizados (Thomas y Gohil, 2011) y son propensos a transportarse con un largo alcance (Lohman et al., 2007), logrando llegar a los cuerpos de agua (Qiu et al., 2004; Tsygankov y Boyarova, 2015), aún a pesar de que el suelo mantiene la función de filtro y almacena estos contaminantes (Weiss et al., 2008). Se ha demostrado la acumulación progresiva del DDD en los animales a través de la cadena trófica (Matache et al., 2016), asimismo diversos estudios han demostrado que 
una larga exposición al DDT y sus metabolitos, aún en dosis bajas, pueden vincularse con efectos adversos sobre la salud humana, tales como apoptosis y genotoxicidad (Díaz-Barriga et al., 2004), supresión inmunológica, disrupción hormonal, disminución de la inteligencia, anomalías reproductivas, cáncer (Gupta y Ali, 2001; Cha et al., 2016), obesidad y diabetes mellitus tipo 2 (Park et al., 2014).

Los pesticidas contienen compuestos químicos que resisten en distintos niveles la degradación y obligan a que cada uno de ellos deba ser tratado de modo particular para su eliminación o reducción (Domene, 2012). Se ha estudiado el tratamiento del DDD en agua a través de la nanofiltración (Pang et al., 2010; Jürgens et al., 2016), con residuos de bagazo de la industria azucarera (Gupta y Ali, 2001), materiales mesoporosos Fe-SBA-15 (Wang et al., 2012), fotodegradación (Ananpattarachai y Kajitvichyanukul 2015) y degradación a través de microorganismos (Carrillo-Pérez et al., 2004; Orozco-Borbón et al., 2008) y hongos (Thomas y Gohil, 2011).

Las zeolitas prometen ser una alternativa para tratar agua contaminada, debido a su porosidad, su estructura tetraédrica tridimensional de $\mathrm{AlO}_{4}$ y $\mathrm{SiO}_{4}$ (Tschernich, 1992) y a su bajo costo (Kehinde y Aziz, 2016). Han tenido una amplia aplicación en el cuidado del ambiente natural (Asgari et al., 2012; Alvarado et al., 2013), aun así, no existe evidencia suficiente del uso de zeolitas con partículas de metales para la eliminación de compuestos orgánicos persistentes (COPs) (Xing et al., 2009). En ese sentido, la Clinoptilolita [(Na, K, Ca) $\left(\mathrm{Al}_{6} \mathrm{Si}_{30} \mathrm{O}_{72}\right) 24 \mathrm{H}_{2} \mathrm{O}$ ( Tsitsishvili et al., 1992) ha ido ganando el interés de los científicos debido a sus características de sorción de substancias orgánicas e inorgánicas (Babel y Kurniawan, 2003; Sprynskyy et al., 2006; Wang y Peng, 2010), tener un volumen de poro del $34 \%$ y una capacidad de intercambio que va desde 0.05 hasta $2.16 \mathrm{meq} / \mathrm{g}$ (Sprynskyy et al., 2006, Wang y Peng, 2010). Por lo anterior, el objetivo de este trabajo es tener un material compuesto por clinoptilolita-plata (CL-Ag) que remueva DDD del agua, ya que es un material que se encuentra en abundancia, disponible y en yacimientos mexicanos (Vaca et al., 2001), que ha sido poco estudiado en su uso de adsorbente de pesticidas, pudiendo ser de gran importancia para las regiones que presentan ese problema de contaminación.

\section{MATERIALES Y MÉTODOS Materiales}

Las sustancias químicas utilizadas fueron adquiridas en la compañía Sigma-Aldrich México: $\mathrm{AgNO}_{3}$ con un $99 \%$ de pureza; hexano con una pureza de $99 \%$ y DDD con pureza de un $99.5 \%$. La clinoptilolita se obtuvo de un yacimiento ubicado geográficamente en las coordenadas $29^{\circ} 31^{\prime}$ de latitud Norte y $110^{\circ} 20^{\prime}$ de longitud Oeste en Ures, Sonora, México.

\section{Preparación de las muestras}

Una muestra de $1 \mathrm{~kg}$ de clinoptilolita se limpió de la suciedad superficial, se trituró con una trituradora de acero, se molió en mortero de porcelana y se tamizó hasta obtener una medida de grano $\leq 0.600 \mathrm{~mm}$. Posteriormente, la muestra fue lavada tres veces con agua desionizada, se colocó en charolas de aluminio y se secó en una estufa Shellab, modelo 1445 a una temperatura de $80^{\circ} \mathrm{C}$ durante 24 horas, a fin de eliminar la humedad.

La modificación de la zeolita se realizó con una solución de nitrato de plata en concentración $9.5 \mathrm{mM}, 19 \mathrm{mM}$ y $28.5 \mathrm{mM}$, para tener clinoptilolita con 1\% de plata (CLIAg1\%), clinoptilolita con $2 \%$ de plata (CLIAg2\%) y clinoptilolita con $3 \%$ de $\mathrm{Ag}(\mathrm{CLIAg} 3 \%)$, éstas se pusieron en un baño marca LabTech, modelo LSB0305 con agitación constante de 100 $\mathrm{rpm}$, a $60^{\circ} \mathrm{C}$ durante $8 \mathrm{~h}$ con una relación de masa/volumen de $0.1 \mathrm{~g} / \mathrm{mL}$.

Al término, las muestras se filtraron por gravedad utilizando papel filtro Whatman número 2 y se colocaron en charolas de aluminio para ser secadas en una estufa a una temperatura de $40^{\circ} \mathrm{C}$ durante 24 horas.

Aparte, se preparó una solución de DDD en una concentración de 1 ppm, se disolvió la sal de DDD en hexano y se llevó a un aforo de $1000 \mathrm{~mL}$ utilizando agua desionizada.

\section{Técnicas de análisis}

El área específica de las muestras se evaluó mediante el método de adsorción de gas nitrógeno, usando un equipo automatizado Belsorp Max Instrument Bel Japan Inc., empleando el método Brunauer-Emmett-Teller (BET).

Para el análisis de la morfología y de la composición química de la CLI en su forma natural y en su forma químicamente modificada con Ag, se usó un Microscopio Electrónico de Barrido (MEB) (JEOL JSM-5410LV), equipado con un sistema INCA y un detector de rayos $\mathrm{X}$ de energía dispersiva (Oxford Instrument), operado con un voltaje de 20 kV. Para el análisis de $M E B$, las muestras se montaron en portamuestras de cobre usando cinta adhesiva de carbono de doble cara. Los especímenes se recubrieron con oro para proporcionar conducción y evitar la carga bajo bombardeo de electrones. La observación de la muestra se realizó bajo alto vacío utilizando el detector de electrones secundarios.

Para determinar la concentración de DDD se utilizó un espectrofotómetro de UV-VIS marca Agilent, modelo 8453. Previamente se realizó la curva de calibrado, utilizando las siguientes concentraciones de $5 \times 10^{-6}, 1 \times 10^{-5}, 3 \times 10^{-5}, 6 \times 10^{-5} \mathrm{y}$ $9 \times 10^{-5} \mathrm{M}$ de DDD.

\section{Remoción de DDD}

Para determinar la cinética de remoción del DDD se pusieron en contacto, en tubos independientes, $5 \mathrm{~mL}$ de solución de DDD en concentración de 1 ppm más $0.025 \mathrm{~g}$ de cada muestra de zeolita (CLI, CLIAg1 \%, CLIA 2\%, CLIAg3\%). Las suspensiones se colocaron en un baño con agitación constante de $100 \mathrm{rpm}$, durante 15, 30, 45, 60, 90, 120, 180 y 360 minutos, a las temperaturas de 25,35 y $45^{\circ} \mathrm{C}$. Al término del tiempo de contacto, las muestras fueron filtradas y la concentración de DDD fue medida en un espectrofotómetro. 
Las isotermas de adsorción se realizaron con $5 \mathrm{~mL}$ de solución de DDD en las siguientes concentraciones: 0.1, $0.2,0.3,0.4,0.5,0.6,0.7,0.8,0.9$, y 1.0 ppm más $0.025 \mathrm{~g}$ de cada muestra de zeolita (CLI, CLIAg1\%, CLIAg2\%, CLIAg3\%). Las suspensiones se colocaron en un baño a una velocidad de agitación constante de $100 \mathrm{rpm}$, durante 180 minutos, tiempo en que se logró el equilibrio de adsorción, a las temperaturas de $25^{\circ} \mathrm{C}, 35^{\circ} \mathrm{C}$ y $45^{\circ} \mathrm{C}$. Al término del tiempo de contacto, las muestras fueron filtradas y la concentración de DDD fue medida en un espectrofotómetro.

La cantidad de DDD adsorbido por la zeolita a un determinado tiempo $\left(q_{t}, \mathrm{mg} / \mathrm{g}\right)$ y en el equilibrio $\left(q_{e}, \mathrm{mg} / \mathrm{g}\right) \mathrm{se}$ calculó utilizando las ecuaciones 1 y 2 :

$$
\begin{aligned}
& q_{t}=\frac{\left(C_{0}-C_{t}\right)}{m} V \\
& q_{e}=\frac{\left(C_{0}-C_{e}\right)}{m} V
\end{aligned}
$$

Donde $C_{0}$ y $C_{e}(\mathrm{mg} / \mathrm{L})$ son la concentración inicial y final del adsorbato, respectivamente. $C_{t}(\mathrm{mg} / \mathrm{L})$ es la concentración a un tiempo $t, V$ es el volumen de la solución (L) y $m$ es la masa del adsorbente (g).

El porcentaje de remoción de DDD (A\%) fue calculado con la ecuación (3).

$$
A \%=\frac{C_{0}-C_{e}}{C_{0}} \times 100
$$

La cinética de adsorción de DDD en los materiales fueron examinados por los modelos de pseudo primer orden y pseudo segundo orden. El primer modelo dado por Lagergren (1898) es definido como:

$$
\frac{d q}{d t}=k_{1}\left(q_{e}-q_{t}\right)
$$

Integrando la ecuación 4 con respecto a las condiciones límites $q=0$ a $t=0$ y $q=q_{e}$ a $t=t$ se obtiene

$$
\log \left(q_{e}-q_{t}\right)=\log \left(q_{e}\right)-\frac{k_{1}}{2.303 t}
$$

La ecuación de pseudo segundo orden basado en la adsorción de equilibrio se expresa como (Ho et al., 1999):

$$
\frac{t}{q_{t}}=\frac{1}{k_{2} q_{e}^{2}}+\frac{t}{q_{e}}
$$

Donde $k_{1}$ es la constante de velocidad de adsorción de Lagergren $\left(\mathrm{min}^{-1}\right), k_{2}(\mathrm{~g} / \mathrm{mg} \mathrm{min})$ es la constante de velocidad de pseudo segundo orden, $q_{t}$ y $q_{e}$ son las cantidades de DDD adsorbido a un tiempo $t$ y en el equilibrio respectivamente, $t$ (min).

Los datos experimentales de equilibrio del intercambio de DDD se ajustaron con el modelo de Langmuir (1916) que se representa con la ecuación siguiente:

$$
q_{e}=\frac{q_{m} b C_{e}}{1+b C_{e}}
$$

Donde $C_{e}$ es la concentración de DDD en el equilibrio, $q_{m}$ y $b$ son los parámetros relacionados con la máxima capacidad de adsorción y la interacción del adsorbato-adsorbente, respectivamente. Las constantes de la isoterma se estimaron utilizando la ecuación linealizada que se expresa:

$$
\frac{C_{e}}{q_{e}}=\frac{1}{q_{m}} C_{e}+\frac{1}{b q_{m}}
$$

\section{Parámetros termodinámicos}

La determinación de los parámetros termodinámicos permite estimar la factibilidad del proceso de adsorción y el efecto de la temperatura en éstos. La ecuación de Van't Hoff, presentada en la fórmula 9, permite obtener de manera gráfica los valores $\Delta \mathrm{H}^{\circ}$ y $\Delta \mathrm{S}^{\circ}$.

$$
\ln K_{c}=-\frac{\Delta H^{o}}{R T}+\frac{\Delta S^{o}}{R}
$$

Un gráfico con $1 / \mathrm{T}$ en el eje de las abscisas y $\ln \mathrm{K}_{c}$ en el eje de las ordenadas, proporciona un intercepto que equivaldría a $\Delta S^{\circ} / R$ y la pendiente sería numéricamente igual a $-\left(\Delta \mathrm{H}^{\circ}\right) / R$, donde $R$ es la constante de los gases ideales (8.314 $\mathrm{J} / \mathrm{mol} \mathrm{K})$. Por otra parte, $K_{c}$ se determina con la ecuación 10.

$$
K_{c}=\frac{C_{A e}}{C_{S e}}
$$

Donde $C_{A e}$ es la concentración del adsorbato en el equilibrio contenido en la superficie del adsorbente y $C_{S e}$ es la concentración en la solución en el equilibrio.

El cambio en la energía libre estándar de Gibbs $\left(\Delta G^{\circ}\right)$ se determina con la ecuación 11:

$$
\Delta G^{o}=-R T \ln K_{c}
$$

\section{RESULTADOS Y DISCUSIÓN}

En la tabla 1 se presentan los resultados del análisis por MEB-EDX realizados a la clinoptilolita mexicana en su forma natural y en su forma modificada identificada como CLIAg3\%. Se aprecia que la clinoptilolita natural al ser tratada con plata incorpora el $2.72 \%$ del peso de ésta, indicando que se produjo un proceso de intercambio iónico, lo que era de esperarse, ya que las zeolitas tienen las propiedades de adsorción, tamiz molecular e intercambio catiónico (Giannetto et al., 2000; Zheng et al., 2017). Los principales cationes de intercambio son $\mathrm{Ca}>\mathrm{Na}<\mathrm{K}$, lo que la hace una clinoptilolita de baja sílice (Jiménez, 2004), que tiene la capacidad de realizar intercambio con el medio externo y modificar la superficie de la zeolita utilizando metales pesados, para que se pueda usar en la adsorción de compuestos inorgánicos (Wang y Peng, 2010). Se muestra que la clinoptilolita en estudio tiene una relación $\mathrm{Si} / \mathrm{Al}$ de alrededor de 4, valor ligeramente inferior a lo presentado por clinoptilolitas de varias partes del mundo (Alpat et al., 2008; Ashrafizadeh et al., 2008; Cabrera et al., 2005; Capasso et al., 2007; Du et al., 2005; Rozic et al., 2000; 
Tabla 1. Resultados por MEB-EDX de CLI y CLIAg3\%.

Table 1. Results by SEM-EDX of CLI and CLIAg3\%.

\begin{tabular}{|c|c|c|c|c|c|c|c|c|c|c|}
\hline \multirow[b]{2}{*}{ Muestra } & \multicolumn{10}{|c|}{ Elemento (\% peso) } \\
\hline & C & 0 & $\mathrm{Na}$ & Mg & Al & Si & $\mathbf{K}$ & $\mathrm{Ca}$ & $\mathrm{Fe}$ & Ag \\
\hline CLI & 7.77 & 55.45 & 0.57 & 0.78 & 5.82 & 25.72 & 1.11 & 2.00 & 0.78 & 0.00 \\
\hline CLIAg3\% & 8.91 & 57.21 & 0.27 & 0.99 & 5.51 & 21.83 & 0.64 & 1.35 & 0.57 & 2.72 \\
\hline
\end{tabular}

Wang y Zhu, 2006), lo que significa que el mineral en estudio tiene una mayor cantidad de sitios de intercambio iónico, ya que la capacidad de intercambio de una zeolita depende de la cantidad de tetraedros de aluminio $\left[\mathrm{AlO}_{4}\right]^{5-}$ que están en el esqueleto estructural de la misma (Leyva-Ramos et al., 2005).

En la figura 1, se muestra la superficie morfológica de la muestra CLI, se observa que tiene placas separadas, no se visualizan granos individuales de cristal de la clinoptilolita, sino agregados del mineral de algunos micrómetros de tamaño, este aspecto es consistente con clinoptilolitas reportadas por Cincotti et al (2006), Hernández et al (2010) y Wang y Peng (2010). También se ve una estructura de superficie irregular con poros y partículas heterogéneos en forma y tamaño, esto se debe a que las zeolitas naturales presentan dos tipos de porosidad: la primaria, atribuible a los microporos y la secundaria, debida a los mesoporos (Hernández et al., 2010), como el diámetro de poro dependerá del número de tetraedros que lo formen y suele estar entre 0.3 y $0.74 \mathrm{~nm}$ (Giannetto et al., 2000), permite que se lleve a cabo la adsorción del ion plata que tiene un radio de hidratación de 0.341 nm (Nightingale, 1959).

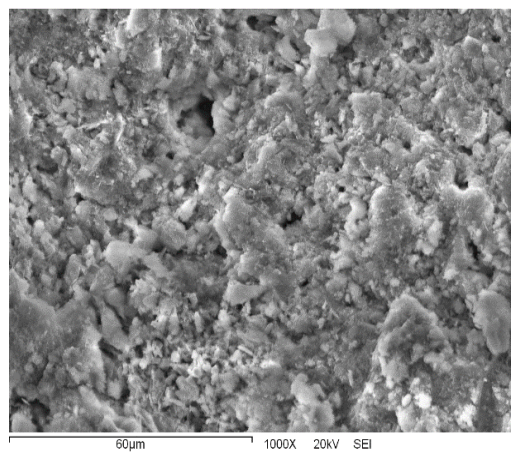

Figura 1. Imagen MEB de la muestra CLI. Figure 1. MEB image of the CLI sample.

En la figura 2, se observa que la superficie morfológica de la muestra CLIAg3\%, está formada por una matriz con algunos cristales visibles y placas aglomeradas del orden de micrómetros, lo cual podemos atribuir a una mezcla de fase microcristalina y una amorfa. Se aprecia una región brillante, lo que corresponde a oclusión de plata en su forma metálica (Leyva-Ramos et al., 2005).

En la tabla 2 se presentan los resultados del análisis determinado por adsorción de gas de nitrógeno (modelo BET) del área específica de las muestras en estudio. Los valores para el área específica de las muestras modificadas decrecen con respecto a la natural, fenómeno que coincide

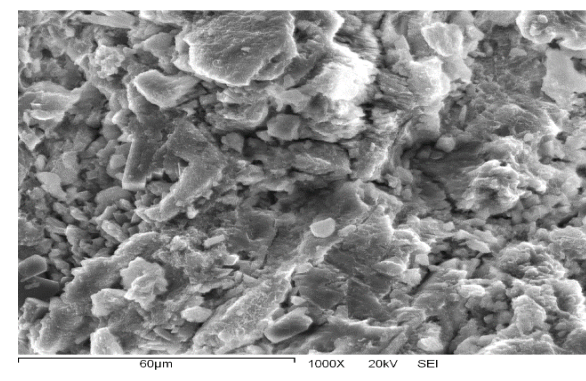

Figura 2. Imagen MEB de la muestra CLIAg3\%. Figure 2. MEB image of the CLIAg3\% sample.

Tabla 2. Valores de área específica para las muestras en estudio.

Table 2. Specific area values for the samples under study.

\begin{tabular}{lc}
\hline Muestras & Área BET $\left(\mathbf{m}^{2} / \mathbf{g}\right)$ \\
\hline CLI & 5.055 \\
CLIAg1\% & 4.6084 \\
CLIAg2\% & 4.8379 \\
CLIAg3\% & 4.9540 \\
\hline
\end{tabular}

con lo reportado por Susuky y Okuhara (2001). El valor de área específica de la muestra CLI es de $5.055 \mathrm{~m}^{2} / \mathrm{gr}$, valor muy cercano a lo reportado por Jiménez-Cedillo y colaboradores (2009) para una clinoptilolita adquirida comercialmente con una empresa mexicana, aunque los valores de área específica que presentan diversos estudios dependen del método seleccionado y de las condiciones experimentales (Ribeiro et al., 2003). Las propiedades de las zeolitas naturales variarán de una localidad a otra dependiendo de su origen y los resultados del área específica se pueden explicar por las áreas de sección transversal de las moléculas de los adsorbatos, ya que los diferentes valores hacen que la accesibilidad dentro de la estructura interna de los poros sea disimil .

\section{Cinéticas de remoción}

En las figuras 3, 4, 5 y 6 se presentan los resultados del efecto del tiempo en la adsorción de DDD, éste se midió en las cuatro muestras de zeolita (CLI, CLIAg1\%, CLIAg2\% y CLIAg3\%), con una dosis de $5 \mathrm{~g} / \mathrm{l}$ a las temperaturas de 25 ${ }^{\circ} \mathrm{C}, 35^{\circ} \mathrm{C}$ y $45^{\circ} \mathrm{C}$. Se aprecia claramente que el equilibrio para el DDD en las cuatro muestras se da a los 180 minutos de tiempo de contacto independientemente de la temperatura y que el comportamiento de la adsorción es similar en todas las muestras.

En las muestras CLI y CLIAg3\%, figuras 3 y 6 respectivamente, se aprecia que la temperatura no tiene efecto en la adsorción de DDD, comparando la remoción en ambas muestras se tiene que la CLI alcanza una adsorción de 0.12 


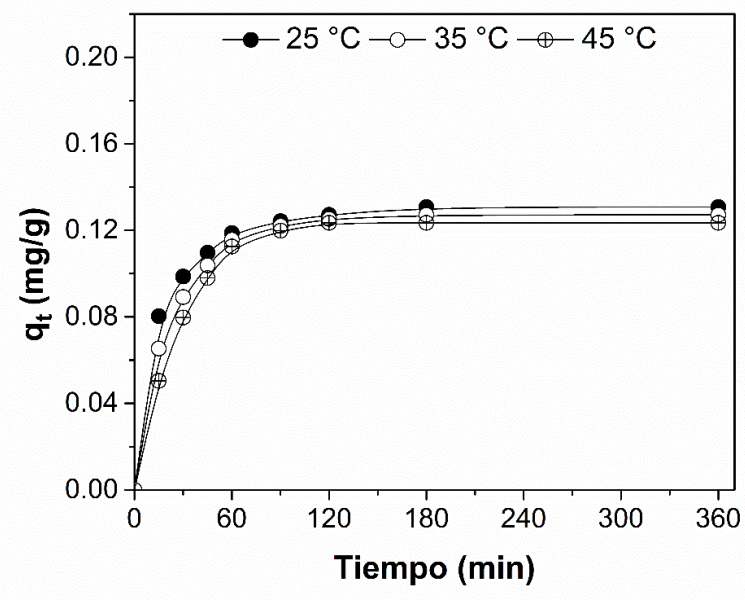

Figura 3. Curvas cinéticas de la adsorción de DDD en CLI a diferentes temperaturas.

Figure 3. Kinetic curves of DDD adsorption in CLI at different temperatures.

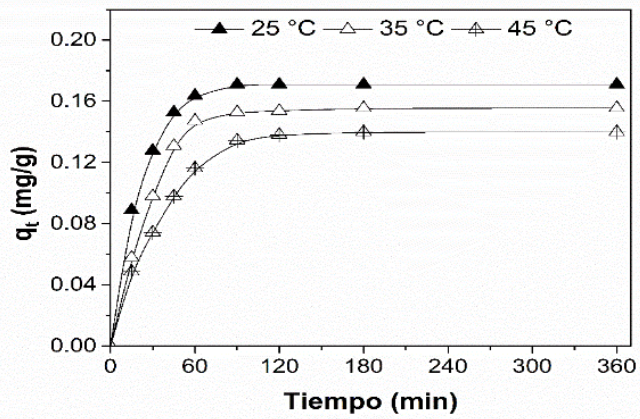

Figura 4. Curvas cinéticas de la adsorción de DDD en CLIAg1\% a diferentes temperaturas.

Figure 4. Kinetic curves of DDD adsorption in CLIAg1\% at different temperatures.

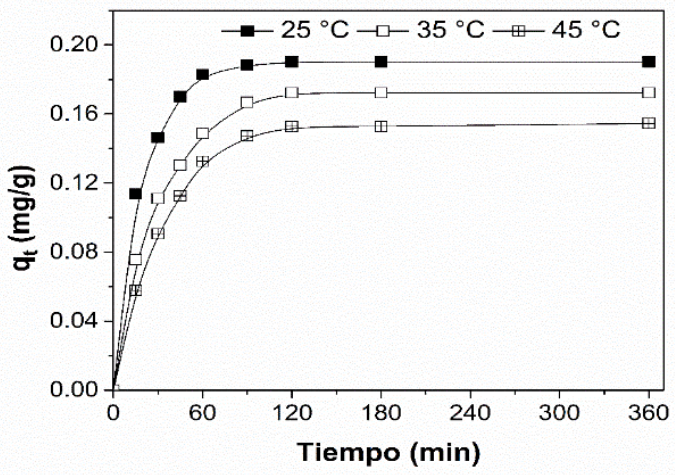

Figura 5. Curvas cinéticas de la adsorción de DDD en CLIAg2\% a diferentes temperaturas.

Figure 5. Kinetic curves of DDD adsorption in CLIAg2\% at different temperatures.

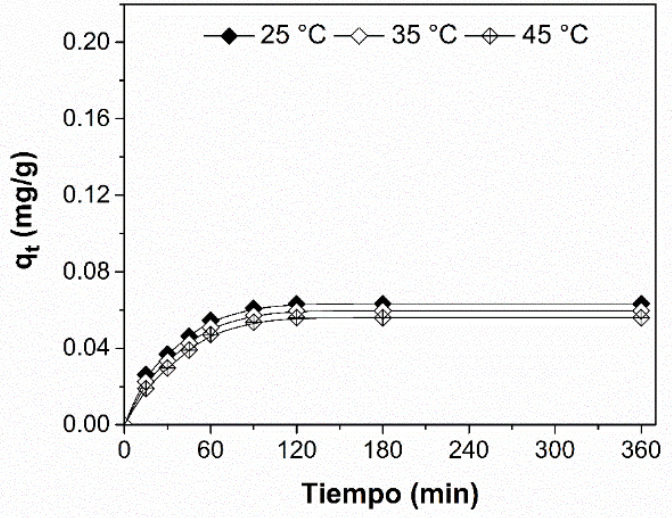

Figura 6. Curvas cinéticas de la adsorción de DDD en CLIAg3\% a diferentes temperaturas.

Figure 6. Kinetic curves of DDD adsorption in CLIAg3\% at different temperatures.

$\mathrm{mg} / \mathrm{g}$ mientras que la CLIAg3\% presenta una adsorción de $0.06 \mathrm{mg} / \mathrm{g}$. Lo anterior se puede explicar ya que se sabe que no todos los sitios catiónicos de una zeolita están disponibles para el intercambio y que los sitios catiónicos localizados en la superficie externa se saturaron con plata y el DDD no pudo penetrar dentro de las cavidades y canales del esqueleto estructural de la clinoptilolita debido a su forma tridimensional, el tamaño molecular por la cantidad de cloros y la aromaticidad.

En las figuras 4 y 5 , correspondientes a las muestras CLIAg1\% y CLIAg2\%, se evidencia que la adsorción de DDD es mayor cuando disminuye la temperatura y que el máximo de adsorción ocurre a los $25^{\circ} \mathrm{C}$, este comportamiento fue reportado por Leyva-Ramos y colaboradores (2005), al encontrar que la capacidad de la clinoptilolita para intercambiar plata incremento 1.5 veces al pasar de $25^{\circ} \mathrm{C}$ a $15^{\circ} \mathrm{C}$. Además, en las figuras 3 y 5 se hace evidente la participación de la plata en la remoción de DDD, logrando $95 \%$ más de remoción la CLIAg2\% (0.19 mg/g) vs. CLI (0.12 mg/g), resultado validado en pruebas por triplicado.

El análisis de los datos de adsorción es importante para el desarrollo de ecuaciones de equilibrio y cinética que puedan ser usadas para propósitos de diseño. La carga de DDD adsorbido por masa de adsorbente se cuantificó mediante los modelos cinéticos de pseudo primer orden y pseudo segundo orden, que han sido ampliamente utilizados para predecir la cinética de adsorción (Murillo et al., 2011).

En la tabla 3 se presentan los parámetros de los modelos cinéticos de pseudo primer orden y pseudo segundo orden para las diferentes muestras estudiadas. Comparando los valores obtenidos experimentalmente para cada una de las muestras con los valores calculados de $q_{e}$ en ambos modelos, se observa una correlación muy cercana con el modelo cinético de pseudo segundo orden, además, los coeficientes de determinación variaron en un intervalo de 0.9933 a 0.9997, lo que indica la aplicabilidad del modelo para des- 
Tabla 3. Parámetros de los modelos cinéticos de pseudo primer orden y pseudo segundo orden para las diferentes muestras estudiadas. Table 3. Parameters of the pseudo first order and pseudo second order kinetic models for the different samples studied.

\begin{tabular}{|c|c|c|c|c|c|c|c|c|c|c|c|c|}
\hline & \multicolumn{3}{|c|}{ CLI } & \multicolumn{3}{|c|}{ CLIAg 1\% } & \multicolumn{3}{|c|}{ CLIAg2\% } & \multicolumn{3}{|c|}{ CLIAg3\% } \\
\hline & $25^{\circ} \mathrm{C}$ & $35^{\circ} \mathrm{C}$ & $45^{\circ} \mathrm{C}$ & $25^{\circ} \mathrm{C}$ & $35^{\circ} \mathrm{C}$ & $45^{\circ} \mathrm{C}$ & $25^{\circ} \mathrm{C}$ & $35^{\circ} \mathrm{C}$ & $45^{\circ} \mathrm{C}$ & $25^{\circ} \mathrm{C}$ & $35^{\circ} \mathrm{C}$ & $45^{\circ} \mathrm{C}$ \\
\hline \multicolumn{13}{|c|}{ Pseudo primer orden } \\
\hline$q_{e}(\mathrm{mg} / \mathrm{g})$ & 0.0730 & 0.1024 & 0.1336 & 0.1999 & 0.1515 & 0.1763 & 0.1824 & 0.1977 & 0.1669 & 0.0770 & 0.0682 & 0.0591 \\
\hline$k_{1}\left(\min ^{-1}\right)$ & 0.0277 & 0.0340 & 0.0398 & 0.0541 & 0.0404 & 0.0371 & 0.0513 & 0.0381 & 0.0338 & 0.0374 & 0.0355 & 0.0299 \\
\hline $\mathrm{R}^{2}$ & 0.9887 & 0.9925 & 0.9947 & 0.9940 & 0.9507 & 0.9856 & 0.9940 & 0.9795 & 0.9937 & 0.9831 & 0.9829 & 0.9834 \\
\hline \multicolumn{13}{|c|}{ Pseudo segundo orden } \\
\hline$q_{e}(\mathrm{mg} / \mathrm{g})$ & 0.1349 & 0.1325 & 0.1302 & 0.1763 & 0.1641 & 0.1517 & 0.1950 & 0.1820 & 0.1652 & 0.0674 & 0.0640 & 0.0607 \\
\hline$k_{2}(\mathrm{~g} / \mathrm{mg} \min )$ & 0.8299 & 0.6956 & 0.5549 & 0.7457 & 0.4391 & 0.3019 & 0.8013 & 0.3756 & 0.3259 & 0.8744 & 0.8189 & 0.7528 \\
\hline $\mathrm{R}^{2}$ & 0.9997 & 0.9989 & 0.9962 & 0.9986 & 0.9947 & 0.9941 & 0.9992 & 0.9974 & 0.9963 & 0.9963 & 0.9966 & 0.9933 \\
\hline
\end{tabular}

cribir la cinética del DDD en el material de clinoptilolita con plata. Esto representa la quimisorción debida a la formación de enlaces químicos entre adsorbente y adsorbato en una monocapa en la superficie (Kavitha y Namasivayam, 2007; Hameed et al., 2008).

Las constantes de velocidad se emplean para calcular la velocidad de adsorción inicial. Según las correlaciones obtenidas con el modelo de pseudo segundo orden, que fue el que se ajustó favorablemente, se determinó una mayor velocidad de adsorción en todos los casos a los $25^{\circ} \mathrm{C}$ y el valor empieza a disminuir cuando se aumenta la temperatura. Esto refuerza lo dicho anteriormente, que cuando aumenta la temperatura hay una mayor interacción entre las moléculas del adsorbato, lo que disminuye el proceso de adsorción. Aunque se utilizó la misma cantidad de adsorbente, este puede no ser suficiente para que las moléculas de DDD sean adsorbidas rápidamente cuando se aumenta la temperatura.

\section{Isotermas de adsorción}

En las figuras 7, 8, 9 y 10 se presentan los resultados del efecto de la temperatura en la adsorción efectuada con diferentes concentraciones de DDD a un tiempo de contacto de 180 minutos. Se muestra que la adsorción va incrementando hasta llegar a un promedio de $0.12 \mathrm{mg} / \mathrm{g}$ y después se vuelve constante indicando que la máxima capacidad de adsorción del adsorbente es de $0.14 \mathrm{mg} / \mathrm{g}$ con una eficiencia de remoción del $95 \%$ para el DDD, con respecto a la muestra testigo (CLI) y el resultado se validó en muestras por triplicado. Se evidencia que la capacidad de adsorción no es influenciada por la temperatura para concentraciones bajas de DDD. Sin embargo, la adsorción disminuye cuando aumenta la temperatura a concentraciones altas. También se observa que la máxima adsorción se da a los $25^{\circ} \mathrm{C}$. En este sentido se encuentra que el orden de adsorción es $25^{\circ} \mathrm{C}>35^{\circ} \mathrm{C}>45^{\circ} \mathrm{C}$. Al tener que la adsorción es menor cuando la temperatura es mayor, se concluye que el proceso es de naturaleza exotérmica (Gupta y Ali, 2010), lo que pudiera deberse a que el DDD posee más energía cuando la temperatura se incrementa, por lo que requieren de más energía para intercambiarse $y$, por ende, al aumentar la temperatura el DDD es removido en menor cantidad.

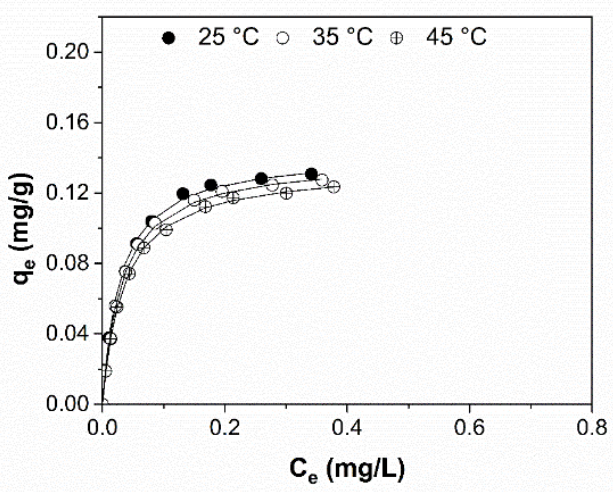

Figura 7. Isoterma de adsorción de DDD en CLI a distintas temperaturas. Figure 7. DDD adsorption isotherm in CLI at different temperatures.

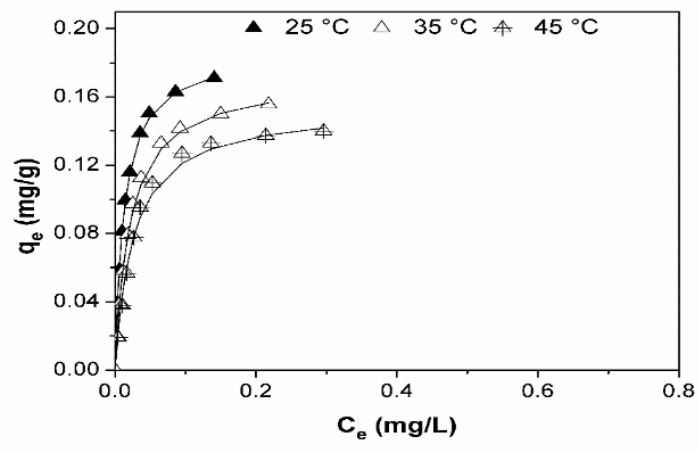

Figura 8. Isoterma de adsorción de DDD en CLIAg1\% a distintas temperaturas.

Figure 8. DDD adsorption isotherm in CLIAg1\% at different temperatures.

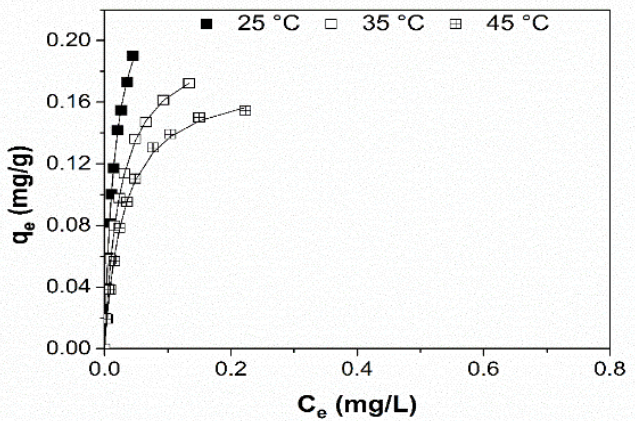

Figura 9. Isoterma de adsorción de DDD en CLIAg2\% a distintas temperaturas.

Figure 9. DDD adsorption isotherm in CLIAg2\% at different temperatures. 


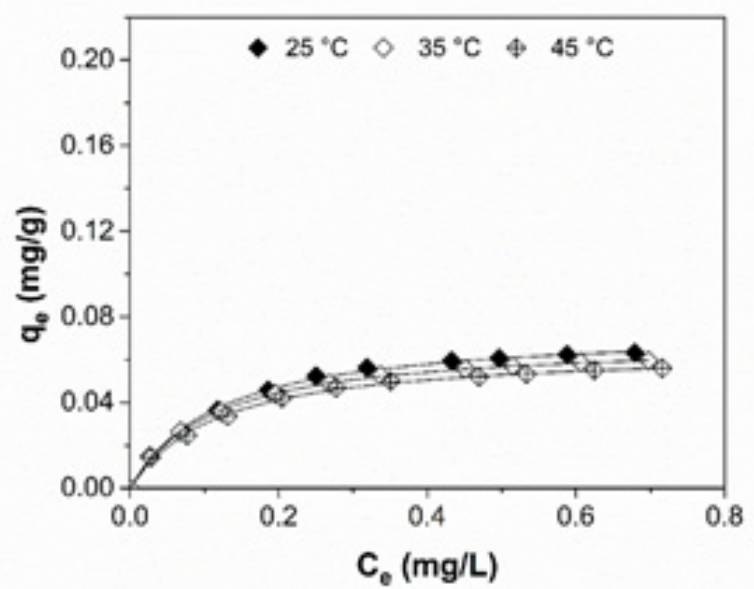

Figura 10. Isoterma de adsorción de DDD en CLIAg3\% a distintas temperaturas.

Figure 10. DDD adsorption isotherm in CLIAg3\% at different temperatures.

Los datos experimentales de equilibrio de la adsorción de DDD se ajustaron con el modelo de Langmuir, similares trabajos han reportado que el equilibrio de intercambio en las zeolitas naturales se puede representar por este modelo (Weber et al., 1983; Al-Haj y El-Bastawi, 1997; Leyva-Ramos et al., 2004; Gupta y Ali, 2010). Esta isoterma interpretó satisfactoriamente los datos experimentales ya que la adsorción depende del número de átomos de aluminio presente en el esqueleto estructural, lo que supone que los cationes se intercambian sobre los sitios catiónicos de la zeolita natural, asume energías de adsorción sobre la superficie y no transmigración de adsorbato en el plano superficial. Las constantes de Langmuir $b$ y $q$ fueron calculadas y los valores obtenidos con las tres diferentes temperaturas se presentan en la tabla 4. Se observa que la constante $b$, relacionada con la energía de adsorción, disminuye cuando hay incremento de la temperatura, lo que indica un decremento de la adsorción a altas temperaturas, teniendo una adsorción exotérmica.

En la tabla 5 se presentan los valores de los parámetros térmicos calculados con los valores experimentales de las isotermas. El valor positivo de $\Delta S^{\circ}$ indica alta posibilidad de reversibilidad; también que significa un aumento en la aleatoriedad en la interfase sólida-solución de iones del DDD durante la adsorción, lo cual también sugiere una buena afinidad de estos iones por el material; por su parte el valor negativo de $\Delta H^{\circ}$ indica que el sistema es exotérmico, y finalmente los valores negativos de los $\Delta G^{\circ}$ obtenidos para cada temperatura, muestran que se tiene un sistema espontáneo, por lo que no es necesario aportar energía al sistema para que este evolucione, los valores son más negativos con el incremento de la temperatura (Bilgin et al., 2013).
Tabla 4. Constantes de Langmuir para el DDD. Table 4. Langmuir constants for DDD.

\begin{tabular}{lcccccc}
\hline Adsorbentes & \multicolumn{6}{c}{ Constantes } \\
\cline { 2 - 7 } & \multicolumn{3}{c}{$\mathbf{q}_{\mathbf{m}} \mathbf{( \mathbf { m g } / \mathbf { g } )}$} & \multicolumn{3}{c}{$\mathbf{b}(\mathbf{L} / \mathbf{m g})$} \\
\cline { 2 - 7 } & $\mathbf{2 5}{ }^{\circ} \mathbf{C}$ & $\mathbf{3 5}{ }^{\circ} \mathbf{C}$ & $\mathbf{4 5}{ }^{\circ} \mathbf{C}$ & $\mathbf{2 5}{ }^{\circ} \mathbf{C}$ & $\mathbf{3 5}{ }^{\circ} \mathbf{C}$ & $\mathbf{4 5}{ }^{\circ} \mathbf{C}$ \\
\hline CLI & 0.1428 & 0.1395 & 0.1348 & 34.0097 & 30.8288 & 28.6605 \\
CLIAg1\% & 0.1877 & 0.1719 & 0.1242 & 75.8960 & 46.7285 & 38.4076 \\
CLIAg2\% & 0.2575 & 0.2048 & 0.1782 & 58.9287 & 39.7364 & 32.5763 \\
CLIAg3\% & 0.0745 & 0.0688 & 0.0648 & 8.7928 & 9.3145 & 8.9231 \\
\hline
\end{tabular}

Tabla 5. Valores termodinámicos para la adsorción de DDD.

Table 5. Thermodynamic values for DDD adsorption.

\begin{tabular}{lcccc}
\hline Muestra & $\begin{array}{c}\text { Temperatura de } \\
\text { estudio }\left({ }^{\circ} \mathbf{C}\right)\end{array}$ & $\begin{array}{c}\Delta \mathbf{G}^{\circ} \\
(\mathbf{k J} / \mathbf{m o l})\end{array}$ & $\begin{array}{c}\Delta \mathbf{H}^{\circ} \\
(\mathbf{k J} / \mathbf{m o l})\end{array}$ & $\begin{array}{c}\Delta \mathbf{S}^{\circ} \\
(\mathbf{J} / \mathbf{m o l})\end{array}$ \\
\hline \multirow{2}{*}{$\mathrm{CLI}$} & 25 & -40.15 & -6.75 & 112.04 \\
& 35 & -41.24 & & \\
\hline CLI1\%Ag & 45 & -42.39 & & \\
& 25 & -42.13 & -26.95 & 50.61 \\
& 35 & -42.31 & & \\
CLI2\%Ag & 45 & -43.16 & & \\
& 25 & -41.51 & -23.43 & 60.44 \\
& 35 & -41.89 & & \\
\hline \multirow{2}{*}{ CLI3\%Ag } & 45 & -42.73 & & \\
& 25 & -36.79 & 0.62 & 125.69 \\
\hline
\end{tabular}

\section{CONCLUSIONES}

La zeolita natural tipo clinoptilolita intercambiada con plata en una concentración de $2 \%$, es efectiva para remover DDD de soluciones acuosas. El DDD es adsorbido a $25^{\circ} \mathrm{C}$ en una concentración máxima de $0.19 \mathrm{mg} / \mathrm{g}$ a los 180 minutos, logrando una remoción del $95 \%$ con respecto a la concentración de origen. La cinética de adsorción del DDD es descrita por el modelo de pseudo segundo orden, representando la quimisorción entre el adsorbato-adsorbente en una monocapa en la superficie. Se encontró que estos procesos son rápidos a menores temperaturas, a mayor temperatura se observa una disminución en la velocidad de adsorción. Los coeficientes de correlación para dicho modelo fueron 0.9933 $-0.9997$.

La remoción es de naturaleza exotérmica y los datos experimentales de las isotermas de adsorción de DDD se ajustaron bien por el modelo de Langmuir. Se encontró que la capacidad de adsorción de la clinoptilolita no es afectada por la temperatura a concentraciones bajas de DDD, pero aumenta a concentraciones altas y cuando la temperatura disminuye, teniendo que el orden de adsorción de los adsorbatos es $25^{\circ} \mathrm{C}>35^{\circ} \mathrm{C}>45^{\circ} \mathrm{C}$. Se deben realizar pruebas de sorción del DDD a temperaturas menores a $25^{\circ} \mathrm{C}$ para determinar la potencialidad de uso de la clinoptilolita en remediación de aguas de uso agrícola en campos mexicanos. 


\section{AGRADECIMIENTOS}

Agradecimiento al Consejo Nacional de Ciencia y Tecnología (CONACyT) por la asignación de beca para los estudios de Maestría en Sustentabilidad de la estudiante Karla Terán Samaniego.

\section{REFERENCIAS}

Al-Haj, A.A. y El-Bastawi, R. 1997. Removal of lead and nickel ions using zeolites tuff. Journal of Chemical Technology and Biotechnology. 69: 27-34.

Alvarado, J., Sotelo, M., Meza, D., Maubert, M. y Paz, F.A. 2013. Evaluación de la Potencialidad de una chabasita natural mexicana en la remoción de plomo en agua. Revista Internacional de Contaminación Ambiental. 29 (2): 201-210.

Alpat, S.K., Özbairak, Ö., Alpat, S. y Akcay, H. 2008. The adsorption kinetics and removal of cationic dye, Toluidine Blue $\mathrm{O}$, from aqueous solution with Turkish zeolite. Journal of Hazardous Materials. 151: 213-220.

Ananpattarachai, J. y Kajitvichyanukul, P. 2015. Photocatalytic degradation of $p, p^{\prime}$-DDT under UV visible light using interstitial $\mathrm{N}$-doped TiO2. Journal of Environmental Science and Health, Part B. 50 (4): 247-260.

Asgari, G., Rahmani, A.R., Askari, F.B. y Godini K. 2012. Catalytic Ozonation of Phenol Using Copper Coated Pumice and Zeolite as Catalysts. Journal of Research in Health Sciences. 12(2): 93-97.

Ashrafizadeh, S.N., Khorasani, Z. y Gorjiara, M. 2008. Ammonia removal from aqueous solutions by Iranian natural zeolite. Separation Science and Technology. 43: 960-978.

ATSDR. Toxicological Profile for DDT, DDE and DDD. Agency for Toxic Substances and Disease Registry. U.S. Department of Health and Human Services. Public Health Service [Consultado 2 junio 2020] 2016. Disponible en: https://www. atsdr.cdc.gov/es/phs/es_phs35.html

Babel, S., y Kurniawan, T.A. 2003. Low-cost adsorbents for heavy metals uptake from contaminated water: a review. Journal of Hazardous Materials 97: 219-243.

Bajwa, A., Ali, V., Mahmood, A., Chaudhry, M.J.I., Syed, J., Li, J., Zhang, G., Jones, K.C. y Malik, R.N. 2016. Organochlorine pesticides (OCPs) in the Indus River catchment area. Pakistan: Status, soil-air exchange and black carbon mediated distribution. Chemosphere.152: 292-300.

Bilgin, E., Özdemir, E. y Beker, U., 2013. Zeolite aupported monoand bimetallic oxides: Promising adsorbentes for removal of $\mathrm{As}(\mathrm{V})$ in aqueous solutions. Chemical Engineering Journal. 220: 402-411.

Cabrera, C., Gabaldon, C., y Marzal, P. 2005. Sorption characteristics of heavy metal ions by a natural zeolite. Journal of Chemical Technology and Biotechnology. 80: 477-481.

Capasso, S., Coppola, E., Iovino, P., Salvestrini, S. y Colella, C. 2007. Sorption of humic acids on zeolitic tuffs. Microporous and Mesoporous Materials. 105: 324-328.

Carrillo-Pérez, E., Ruiz-Manríquez, A. y Yeomans-Reina, H. 2004. Aislamiento, identificación y evaluación de un cultivo mixto de microorganismos con capacidad para degradar DDT. Revista Internacional de Contaminación Ambiental. 20(2): 69-75.

Castro, J. y Díaz, M.L. 2004. Avances del convenio de Estocolmo en México. Las sustancias tóxicas persistentes en México.
Comp. Adrián Fernández Bremauntz, Mario Yarto Ramírez y José Castro Díaz. Secretaría de Medio Ambiente y Recursos

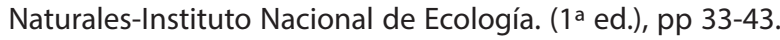
México.

Cha, K.M., Lee, E.S., Kim, I.W., Cho, H.K., Ryu, J.H. y Kim, S.K. 2016. Canola oil is an excellent vehicle for eliminating pesticide residues in aqueous ginseng extract. Journal of Ginseng Research 40(3): 292-299.

Cincotti, A., Mameli, A., Locci, A.M., Orru, R.y Cao, G.H. 2006. Heavy metals uptake by sardinian natural zeolites: experiment and modeling. Industrial and Engineering Chemistry Research. 45: 1074-1084.

Díaz-Barriga, F., López, D., Pérez, I.N., Batres, L.E. y Yáñez, L. 2004. Evaluación del riesgo para las sustancias tóxicas persistentes. Las sustancias tóxicas persistentes en México. Comp. Adrián Fernández Bremauntz, Mario Yarto Ramírez y José Castro Díaz. Secretaría de Medio Ambiente y Recursos NaturalesInstituto Nacional de Ecología. (1 a ed.). pp 233-243. México.

Domene M. Contaminantes Orgánicos Persistentes (COPs), la nocividad que no cesa. Seguridad y Salud Laboral. [Consultado 2 junio 2020] 2012. Disponible en: http:// archivosseguridadlaboral-manueldomene.blogspot. com/2012/01/contaminantes-organicos-persistentes.html

Du, Q., Liu, S., Cao, Z. y Wang, Y. 2005. Ammonia removal from aqueous solution using natural Chinese clinoptilolite. Separation and Purification Technology. 44: 229-234.

Gaspar, F.W., Chevrier, J., Bornman, R., Crause, M., Obida, M., Barr, D.B., Bradman, A., Bouwman, H. y Eskenazi, B. 2016. Corrigendum "Undisturbed dust as a metric of long-term indoor insecticide exposure: Residential DDT contamination from indoor residual spraying and its association with serum levels in the VHEMBE cohort" [Environ. Int. 85C (2015) 163167]. Environmental International. 94: 778-783.

Giannetto, G.P., Montes, A.R. y Rodríguez, G.F. 2000. Zeolitas: características, propiedades y aplicaciones industriales. Innovación Tecnológica. Caracas.

Gupta, V.K. y Ali, I. 2001. Removal of DDD and DDE from wastewater using bagasse fly ash, a sugar industry waste. Water Research. 35: 33-40.

Hameed, B.H., Tan, I.A.W., Ahmad, A.L. 2008. Adsorption isotherm, kinetic modeling and mechanism of 2,4,6-trichlorophenol on coconut husk-based activated carbon. Chemical Engineering Journal. 144(2): 235-344.

Hernández, M.A., Rojas, F., Lara, V.H., Portillo, R., Castelán R., Pérez G. y Salas, R. 2010. Estructura porosa y propiedades estructurales de mordenita y clinoptilolita. Superficies y Vacío. 23(S): 51-56.

Ho, Y.S. y McKay, G. 1999. Pseudo-second order model for sorption processes. Process Biochem. 34: 451-465.

Jiménez, M.J. 2004. Caracterización de Minerales Zeolíticos mexicanos. Tesis para obtener el título de ingeniero químico. Universidad Autónoma del Estado de México.

Jiménez-Cedillo, M., Olguín, M. y Fall, C. 2009. Adsorption kinetic of arsenates as water pollutant on iron, manganese and iron-manganese-modified clinoptilolite-rich tuffs. Journal of Hazardous Materials. 163(2-3): 939-945.

Jürgens, M.D., Crosse, J., Hamilton, P.B., Johnson, A.C. y Jones, K.C. 2016. The long shadow of our chemical past - High DDT concentrations in fish near a former agrochemical factory in England. Chemosphere. 162: 333-344. 
Kavitha, D. y Namasivayam, C. 2007. Experimental and kinetic studies on methylene blue adsorption by coir pith carbon. Bioresource Technology. 98(1):14-21.

Kehinde, F.O. y Aziz, H.A. 2016. Classical optimization of process variables in the treatment of real textile wastewater using clinoptilolita. Journal of Environmental Chemical Engineering. 4: 1242-1247.

Lagergren, S.Y. 1898. Zur Theorie der sogenannten Adsorption gelöster Stoffe. Handlingar, 24(4): 1-39.

Langmuir, I. 1916. The constitution and fundamental properties of solids and liquids. part I solids. Journal of the American Chemical Society. 38: 2221-2295.

Leyva-Ramos, R., Aguilar-Armenta, G., González-Gutiérrez, L., Guerrero-Coronado, R.M. y Mendoza Barrón, J. 2004. Ammonia exchange on clinoptilolite from mineral deposits located in Mexico. Journal of Chemical Technology and Biotechnology. 79: 651-657.

Leyva-Ramos, R., Medellin-Castillo, N.A., Guerrero-Coronado, R.M., Berber Mendoza, M.S., Aragón-Piña A. y Jacobo-Azuara, A. 2005. Intercambio iónico de plata (I) en solución acuosa sobre clinoptilolita. Revista Internacional de Contaminación Ambiental. 21(4): 193-200.

Lohman, R., Breivik, K., Dachs, J. y Muir, D. 2007. Global fate of POPs: current and future research directions. Environmental Pollution. 150(1): 150-165.

Matache, M.L., Hura, C. y David, I.G. 2016. Non-invasive monitoring of organohalogen compounds in eggshells and feathers of birds from the Lower Prut Floodplain Natural Park in Romania. Procedia Environmental Sciences. 32: 49-58.

Murillo, Y.S., Giraldo, L. y Moreno, J.C. 2011. Determinación de la cinética de adsorción de 2,4-dinitrofenol en carbonizado de hueso bovino por espectrofotometría UV-VIS. Revista Colombiana de Química. 40(1): 91-103.

Nightingale, E.R. 1959. Phenomenological theory of ion solvation. Effective radii of hydrated ions. Journal of Physical Chemistry. 63(9): 1381-1387.

Orozco-Borbón, M.V., de la Rosa-Vélez, J., Ramírez-Álvarez, N., Macías-Zamora, V., Gutiérrez-Galindo, E.A. y Muñoz-Barbosa, A. 2008. DDT en sedimentos de la costa noroccidental de Baja California (México) y su biotransformación por Vibrio sp. Ciencias Marinas. 34(4): 419-432.

Pang, W., Gao, N.Y. y Xia, S. 2010. Removal of DDT in drinking water using nanofiltration process. Desalination. 250: 553556.

Park, J.H., Cha, E.S., Ko, Y., Hwang, M.S., Hong, J.H. y Lee, W.J. 2014. Exposure to Dichlorodiphenyltrichloroethane and the Risk of Breast Cancer: A Systematic Review and Meta-analysis. Osong Public Health Research Perspectives. 5 (2): 77-84.

Polanco, A.G., Navarro, J.A., Solorio, J., Mena, G.J., Marrufo, J. y Del Valls, T.A. 2015. Contamination by organochlorine pesticides in the aquifer of the Ring of cenotes in Yucatán, México. Water and Environmental Journal. 29: 140-150.

Qiu, X., Zhu, T., Li, J., Pan, H., Li, Q., Miao, G. y Gong, J. 2004. Organochlorine pesticides in the air around the Taihu Lake, China. Environmental Science and Technology. 38(5): 13681374.
Ribeiro, T., Rubio, J. y Smith, R., 2003. A dried hydrophobic aquaphyte as on oil filter oil/water emulsions. Spill Science and Technology Bulletin. 8(5-6): 483-489.

Rozic, M., Cerjan-Stefanovic, S., Kurajica, S., Vancina, V. y Hodzic, E. 2000. Ammoniacal nitrogen removal from water by treatment with clays and zeolites. Water Research. 34: 36753681.

Sprynskyy, M., Buszewski, B., Terzyk, A.P. y Namiesnik, J. 2006. Study of the selection mechanism of heavy metal $\left(\mathrm{Pb}^{2+}, \mathrm{Cu}^{2+}\right.$, $\mathrm{Ni}^{2+}$ and $\left.\mathrm{Cd}^{2+}\right)$ adsorption on clinoptilolite. Journal of Colloid and Interface Science. 304(1): 21-28.

Suzuki, T. y Okuhara, T. 2001. Change in pore structure of MFI zeolite by treatment with $\mathrm{NaOH}$ aqueous solution. Microporous and Mesoporous Materials. 43(1): 83-89.

Thomas, J.E. y Gohil, H. 2011. Microcosm studies on the degradation of $o, p^{\prime}$-and p, $p^{\prime}$-DDT, DDE and DDD in a muck soil. World Journal of Microbiology and Biotechnology. 27(3): 619-625.

Tschernich, R.W. 1992. Zeolites of the World. 1st ed. Geoscience Press, Inc., Phoenix, AZ.

Tsitsishvili, G.V., Andronikashvili, T.G., Kirov, G.N. y Filizova, L.D. 1992. Natural Zeolites. Ellis Horwood. Chichester.

Tsygankov, V.Y. y Boyarova, M.D. 2015. Sample Preparation Method for the Determination of Organochlorine Pesticides in Aquatic Organisms by Gas Chromatography. Achievements in the Life Sciences. 9: 65-68.

Turusov, V., Rakitsky, V. y Tomatis, L. 2002. Dichlorodiphenyltrichloroethane (DDT): ubiquity, persistence and risks. Environmental Health Perspectives. 110(2): 125-128.

Vaca, M., López, R., Gehr, R., Jiménez, B.E. y Álvarez, P. 2001. Heavy metal removal with mexican clinoptilolite: multicomponent ionic Exchange. Water Research. 35(2): 373-378.

Venier, M., e Hites, R.A. 2014. DDT and HCH, two discontinued organochlorine insecticides in the Great Lakes region: Isomer trends and sources. Environmental International 69:159-165.

Wang, H., Tian, H. y Hao, Z. 2012. Study of DDT and its derivatives DDD, DDE adsorption and degradation over Fe-SBA-15 at low temperature. Journal of Environmental Sciences 24(3): 536-540.

Wang, S. y Peng, Y. 2010. Natural zeolites as effective adsorbents in water and wastewater treatment. Chemical Engineering Journal. 156(1): 11-24.

Wang, S.B. y Zhu, Z.H. 2006. Characterisation and environmental application of an Australian natural zeolite for basic dye removal from aqueous solution. Journal of Hazardous Materials. 136: 946-952.

Weber, M.A., Barbaruck, K.A. y Westfall, D.G. 1983. Ammonium adsorption by a zeolite in a static and dynamic system. Journal of Environmental Quality. 12(4): 549-552.

Weiss, P.T., LeFevre, G. y Gulliver, J.S. Contamination of soil and Groundwater Due to Stormwater infiltration Practices. [Consultado 5 junio 2020] 2008. Disponible en:

https://pdfs.semanticscholar.org/702d/527bbb1b622f44 $8691 d 016 a b 5 b c 5657941 f 2 . p d f ?$ ga $=2.215329623$. 1108367914.1591409512-190085627. 1591409512 
Xing, Y.N., Guo, Y., Xie, M., Shen, R.L. y Zeng, E.Y. 2009. Detection of DDT and its metabolites in two estuaries of South China using a SPME-based device: first report of $p, p^{\prime}$-DDMU in water column. Environmental Pollution 157: 1382-1387.

Zheng, Y., Walker, H. and Zhu, Q. 2017. Reduction of nitrate by $\mathrm{Na} \mathrm{Y}$ zeolite supported $\mathrm{Fe}, \mathrm{Cu} / \mathrm{Fe}$ and $\mathrm{Mn} / \mathrm{Fe}$ nanoparticles. Journal of Hazardous Materials. 324: 605-616.
Ziv, A. 2009. Biodegradación de compuestos orgánicos persistentes (COP): I. El caso de los bifenilos policlorados (PCB). Acta Biológica Colombiana 14(1): 57-88.

Zhou, Q., Wang, J., Meng, B., Cheng, J., Lin, G., Chen, J., Zhen, D. y Yu, Y. 2013. Distribution and sources of organochlorine pesticides in agricultural soils from central China. Ecotoxicology and Environmental Safety. 93: 163-170. 